\title{
A Note on the Justification of the Eddy Current Model in Electrodynamics.
}

\author{
Dirk Pauly* \& Rainer Picard ${ }^{\dagger}$
}

June 3, 2016

\begin{abstract}
The issue of justifying the eddy current approximation of Maxwell's equations is re-considered in the time-dependent setting. Convergence of the solution operators is shown in the sense of strong operator limits.
\end{abstract}

Keywords and phrases: electrodynamics, eddy current problem, evolutionary equations, material laws Mathematics subject classification 2010: 35Q61, 78A25, 78A48

June 3, 2016

\section{Introduction}

Somewhat simplified, the topic of this paper is the study of the limit $\varepsilon \rightarrow 0+$ in the standard Maxwell system in a non-empty open set $\Omega \subseteq \mathbb{R}^{3}$, which in convenient block operator matrix notation is given by

$$
\left(\partial_{0}\left(\begin{array}{ll}
\varepsilon & 0 \\
0 & \mu
\end{array}\right)+\left(\begin{array}{ll}
\sigma & 0 \\
0 & 0
\end{array}\right)+\left(\begin{array}{cc}
0 & -\operatorname{curl} \\
\operatorname{courl} & 0
\end{array}\right)\right)\left(\begin{array}{c}
E \\
H
\end{array}\right)=\left(\begin{array}{c}
-J \\
K
\end{array}\right)
$$

with the limit case $\varepsilon=0$ being the so-called eddy current case. Here, following standard physics notation $\partial_{0}$ denotes the time derivative, $E, H$, denote the electric and magnetic field, respectively, and $J, K$ corresponding external source terms, $\varepsilon$ denotes the dielectricity and $\mu$ the magnetic permeability, $\sigma$ denotes the conductivity. The overset circle in curl is supposed to indicate that the so-called electric boundary condition is imposed on $E$, which makes $\left(\begin{array}{cc}0 & - \text { curl } \\ \text { curl } & 0\end{array}\right)$ skew-selfadjoint in $L^{2}\left(\Omega, \mathbb{C}^{3} \times \mathbb{C}^{3}\right) \equiv L^{2}\left(\Omega, \mathbb{C}^{6}\right)$. For the purpose of this introduction we may think of $\varepsilon, \mu, \sigma$ simply as non-negative real numbers. The approximation question $\varepsilon \rightarrow 0+$ has been considered in the literature commonly in the second order form, where $H$ has been eliminated from the equations, i.e. the equations discussed are not Maxwell's equations but rather the abstract wave equation

$$
\varepsilon \partial_{0}^{2} E+\sigma \partial_{0} E+\operatorname{curl} \mu^{-1} \operatorname{corl} E=-\partial_{0} J+\operatorname{curl} \mu^{-1} K=:-\widetilde{J} .
$$

\footnotetext{
*Fakultät für Mathematik, Universität Duisburg-Essen, Campus Essen, Germany, dirk.pauly@uni-due.de

${ }^{\dagger}$ Institut für Analysis, Technische Universität Dresden, Germany, rainer.picard@tu-dresden.de
} 
Following common wisdom indeed a time-harmonic regime is assumed, where $\partial_{0}$ is replaces by $i \omega$, where $\omega$ is a real number referred to as frequency, leading to

$$
\left(\varepsilon \omega^{2}-\mathrm{i} \omega \sigma\right) E-\operatorname{curl} \mu^{-1} \operatorname{corl} E=\widetilde{J} .
$$

Due to the selfadjointness of $\operatorname{curl} \mu^{-1} \operatorname{corl}$ in $L^{2}\left(\Omega, \mathbb{C}^{3}\right)$ we have that for $\sigma \neq 0$ the number $\varepsilon \omega^{2}-\mathrm{i} \omega \sigma$ is actually in the resolvent set of $\operatorname{curl} \mu^{-1}$ curl and so the limit $\varepsilon \rightarrow 0+$ is well controlled by the analyticity of the resolvent

$$
z \mapsto\left(z-\operatorname{curl} \mu^{-1} \text { currl }\right)^{-1}
$$

on $\mathbb{C} \backslash[0, \infty[$. The situation is less clear if $\varepsilon, \sigma$ are allowed to vary - say they are piece-wise constant. For example there may be a decomposition of $\Omega$ into a relative compact, non-empty, open subset $\Omega_{c} \subseteq \Omega$, where $\sigma=\sigma_{c}>0$ and $\varepsilon=\varepsilon_{c} \geq 0$, and the rest, where $\sigma=0$ and $\varepsilon>0$. For bounded and sufficiently regular domains $\Omega$ such that a suitable compact embedding result holds, the limit $\varepsilon_{c} \rightarrow 0+$ can still be established and so a justification of the eddy current problem can be given. For a survey see [1] and the literature quoted there.

A dramatically different situation arises if $\Omega$ is unbounded, e.g. $\Omega=\mathbb{R}^{3}$. Then $\sigma=0$ becomes the dominant case with the material behavior in $\Omega_{c}$ just being a compact perturbation. In this situation

$$
\varepsilon \omega^{2} E-\operatorname{curl} \mu^{-1} \operatorname{curl} E=\widetilde{J}
$$

is our reference case, where now $\varepsilon \omega^{2} \in \mathbb{R} \backslash\{0\}$ is always in the continuous spectrum of the operator curl $\mu^{-1}$ curl. Thus in contrast to what seems to be claimed in [2] a solution theory in $L^{2}\left(\Omega, \mathbb{C}^{3}\right)$ is unavailable. The much more demanding issues involved to study such perturbation problems and to discuss limiting problems is well developed in connection with the solution theory for exterior boundary value problems and the study of low-frequency asymptotics in e.g. [7], [10, 11, 12, [4, 5], 6], [3]. A comparison between the low-frequency asymptotics for the full time-harmonic Maxwell's equations and their eddy current approximation can be found in [6, Kapitel 5, Satz 5.7].

On the other hand, keeping in mind that time-harmonic problems are non-physical in so far as they produce infinite energy solutions and merely serve to describe the time-asymptotic behavior in presence of a - perpetual - time-harmonic forcing, it seems appropriate to by-pass the above spectral issues altogether by discussing the original - physical - dynamic system directly. This is the perspective of the following presentation, which is based on concepts derived in e.g. [8, 9]. After a brief introduction into the needed framework we discuss the limit to the eddy current case in full generality in section 2. In particular, we emphasize that size and boundary regularity of the underlying domain $\Omega$ play no role in the final result. This is due to the fact that the classical boundary trace results are superfluous for the basic solution theory and for obtaining the convergence result.

\section{The Functionalanalytical Framework}

Key to the approach presented here is to consider the closure of differentiation acting on $C_{1}(\mathbb{R}, H)$-functions with compact support, i.e. functions in $\stackrel{\circ}{C}_{1}(\mathbb{R}, H)$, as an operator in $H_{\varrho}(\mathbb{R}, H)$ with $\left.\varrho \in\right] 0, \infty\left[\right.$, a weighted $L^{2}$-type space with inner product

$$
\langle\varphi \mid \psi\rangle_{\varrho}:=\int_{\mathbb{R}}\langle\varphi(t) \mid \psi(t)\rangle_{H} \exp (-2 \varrho t) d t,
$$


where $\langle\cdot \mid \cdot\rangle_{H}$ denotes the inner product of the Hilbert space $H$. The resulting operator

$$
\partial_{0}: D\left(\partial_{0}\right) \subseteq H_{\varrho}(\mathbb{R}, H) \rightarrow H_{\varrho}(\mathbb{R}, H)
$$

turns out, [8, 9], to be normal with

$$
\mathfrak{R e} \partial_{0}=\varrho .
$$

This observation implies that for bounded linear operators $M_{0}: H \rightarrow H$ and $M_{1}: H \rightarrow H$, where $M_{0}$ is selfadjoint, and for a skew-selfadjoint linear operator $A: D(A) \subseteq H \rightarrow H$, which is possibly unbounded, the relation

$$
\mathfrak{R e}\left\langle u \mid\left(\partial_{0} M_{0}+M_{1}+A\right) u\right\rangle_{\varrho}=\left\langle u \mid\left(\varrho M_{0}+\mathfrak{R e} M_{1}\right) u\right\rangle_{\varrho}
$$

holds for all $u \in D\left(\partial_{0}\right) \cap D(A)$. With the assumption that

$$
\varrho M_{0}+\mathfrak{R e} M_{1} \geq c>0
$$

for all sufficiently large $\varrho \in] 0, \infty\left[\right.$, we obtain that the closure $\overline{\partial_{0} M_{0}+M_{1}+A}$ and its adjoint $\left(\partial_{0} M_{0}+M_{1}+A\right)^{*}=\overline{\partial_{0} M_{0}+M_{1}^{*}-A}$ both have continuous inverses bounded by $1 / c$. In particular, the null spaces of $\overline{\partial_{0} M_{0}+M_{1}+A}$ and $\left(\partial_{0} M_{0}+M_{1}+A\right)^{*}$ are both trivial. Thus, we have the following well-posedness result, see e.g. [8, 9].

Theorem 1.1. Let $M_{k}: H \rightarrow H, k=0,1$, be continuous linear operators, $M_{0}$ selfadjoint, such that (1.2) holds for some $c \in] 0, \infty[$ and for all $\varrho \in] \varrho_{0}, \infty\left[\right.$ with $\left.\varrho_{0} \in\right] 0, \infty[$ sufficiently large. Moreover let $A: D(A) \subseteq H \rightarrow H$ be skew-selfadjoint. Then

$$
\left(\overline{\partial_{0} M_{0}+M_{1}+A}\right) u=f
$$

has for any $f \in H_{\varrho}(\mathbb{R}, H)$ a unique solution $u \in H_{\varrho}(\mathbb{R}, H)$. Furthermore, $u$ depends on $f$ continuously, i.e.

$$
\left(\overline{\partial_{0} M_{0}+M_{1}+A}\right)^{-1}: H_{\varrho}(\mathbb{R}, H) \rightarrow H_{\varrho}(\mathbb{R}, H)
$$

is a continuous linear operator for $\varrho \in] \varrho_{0}, \infty[$.

As a refinement of (1.1) we also find by integration by parts that for $u \in \stackrel{C}{C}_{1}(\mathbb{R}, H)$ (and so for $\left.u \in D\left(\overline{\partial_{0} M_{0}+M_{1}+A}\right)\right)$

$$
\mathfrak{R e}\left\langle u \mid \chi_{]-\infty, a]}\left(\partial_{0} M_{0}+M_{1}\right) u\right\rangle_{\varrho} \geq c\left\langle\chi_{]-\infty, a]} u \mid \chi_{]-\infty, a]} u\right\rangle_{\varrho} .
$$

This yields that we have also causality in the sense of the following theorem.

Theorem 1.2. [Causality] Under the assumptions of Theorem 1.1 we have

$$
\chi_{]-\infty, a]}\left(\overline{\partial_{0} M_{0}+M_{1}+A}\right)^{-1}=\chi_{]-\infty, a]}\left(\overline{\partial_{0} M_{0}+M_{1}+A}\right)^{-1} \chi_{]-\infty, a]}
$$

for all sufficiently large $\varrho \in] 0, \infty[$.

We plan to approach the eddy current approximation within this abstract framework, which simplifies matters in so far as we can deal with the time-dependent situation under very general assumptions on the coefficients, which can indeed be operators acting in the underlying spatial Hilbert space. 


\section{Convergence to the Eddy Current Model}

\subsection{Maxwell's Equations with General Material Laws}

Maxwell's equations with a general, simple material law read:

$$
\left(\partial_{0} M+N+A\right)\left(\begin{array}{c}
E \\
H
\end{array}\right)=\left(\begin{array}{c}
-J \\
K
\end{array}\right), \quad A:=\left(\begin{array}{cc}
0 & - \text { curl } \\
\text { curl } & 0
\end{array}\right) .
$$

Here curl is defined as the closure in $L^{2}\left(\Omega, \mathbb{C}^{3}\right)$ of the classical vectoranalytic operation curl on $C_{1}\left(\Omega, \mathbb{C}^{3}\right)$-vector fields with compact support in the non-empty open set $\Omega \subseteq \mathbb{R}^{3}$, which is obviously symmetric in $L^{2}\left(\Omega, \mathbb{C}^{3}\right)$ and therefore indeed closable. We define

$$
\operatorname{curl}:=\operatorname{corrl}^{*} \text {, }
$$

which is nothing but the classical weak $L^{2}\left(\Omega, \mathbb{C}^{3}\right)$-curl. Due to the structure of $A$ as

$$
A=\left(\begin{array}{cc}
0 & - \text { curl }^{*} \\
\text { curl } & 0
\end{array}\right)
$$

we read off that $A$ is skew-selfadjoint. Since every closed, linear operator gives rise to a canonical Hilbert space by equipping its domain with the graph inner product, we have Hilbert spaces

$$
H(\operatorname{coirl}), H(\operatorname{curl})
$$

from the respective domains $D$ (curl), $D$ (curl). One frequently finds already $H$ (curl) defined in terms of boundary traces, which unnecessarily limits the applicability of the results. Even worse, it suggests to the uninitiate or confused reader that boundary regularity is required to ensure that the physical model actually works. We note that since

$$
\operatorname{curl}=\operatorname{curl}^{*} \subseteq \operatorname{curl}
$$

we have for $E \in H$ (curl), with $\langle\cdot \mid \cdot\rangle_{L^{2}}$ denoting the inner product of $L^{2}\left(\Omega, \mathbb{C}^{3}\right)$, that

$$
\langle E \mid \operatorname{curl} \Psi\rangle_{L^{2}}=\langle\operatorname{coirl} E \mid \Psi\rangle_{L^{2}}=\langle\operatorname{curl} E \mid \Psi\rangle_{L^{2}}
$$

for all $\Phi \in H$ (curl). We read off that conversely

$$
\langle E \mid \operatorname{curl} \Psi\rangle_{L^{2}}=\langle\operatorname{curl} E \mid \Psi\rangle_{L^{2}} \quad \text { for all } \Psi \in H(\operatorname{curl})
$$

characterizes $E \in H$ (curl). This shows that

$$
E \in H(\text { coirl })
$$

is a suitable generalization of the electric boundary condition for the topological boundary of arbitrary non-empty open sets.

According to the above abstract framework, the solvability constraint on the operator coefficients $M=M_{0}, N=M_{1}$ is

$$
\varrho M+\mathfrak{R e} N \geq c>0
$$

for some real constant $c$ and all sufficiently large $\varrho \in] 0, \infty[$. The underlying Hilbert space is $H=L^{2}\left(\Omega, \mathbb{C}^{6}\right)$. We recall that causality of the solution operator is also implied by our general framework. 


\subsection{Classical Electrodynamics and the Eddy Current Problem}

On this basis we are now able to discuss the limiting behavior to the eddy current case. Let

$$
M_{s}:=\left(\begin{array}{cc}
\varepsilon_{s} & 0 \\
0 & \mu_{s}
\end{array}\right), \quad N_{s}:=\left(\begin{array}{cc}
\sigma_{s} & 0 \\
0 & 0
\end{array}\right), \quad s \in[0,1[.
$$

Assuming that for some $\widehat{\varrho} \in] 0, \infty[$ we have for all $\varrho \in] \widehat{\varrho}, \infty[$ and all $s \in[0,1[$

$$
\varrho M_{s}+N_{s} \geq c>0
$$

we have uniform boundedness for the solution operators in the sense that

$$
\left\|\left(\partial_{0} M_{s}+N_{s}+A\right)^{-1}\right\| \leq \frac{1}{c}
$$

for $s \in[0,1[$. On the other hand, we have the following resolvent equation type result for the solution operators:

$$
\begin{aligned}
& \left(\partial_{0} M_{s}+N_{s}+A\right)^{-1}-\left(\partial_{0} M_{0}+N_{0}+A\right)^{-1} \\
= & \left(\partial_{0} M_{s}+N_{s}+A\right)^{-1}\left(\left(M_{0}-M_{s}\right) \partial_{0}+N_{0}-N_{s}\right)\left(\partial_{0} M_{0}+N_{0}+A\right)^{-1}
\end{aligned}
$$

If now

$$
M_{s} \stackrel{s \rightarrow 0+}{\longrightarrow} M_{0}, \quad N_{s} \stackrel{s \rightarrow 0+}{\longrightarrow} N_{0} \quad \text { strongly in } L^{2}\left(\Omega, \mathbb{C}^{6}\right),
$$

we read off that we have

$$
\left(\partial_{0} M_{s}+N_{s}+A\right)^{-1} F \stackrel{s \rightarrow 0+}{\longrightarrow}\left(\partial_{0} M_{0}+N_{0}+A\right)^{-1} F \quad \text { for every } F \in D\left(\partial_{0}\right) .
$$

Due to the uniform boundedness of the solution operators, however, we can use the density of $D\left(\partial_{0}\right)$ in $H_{\varrho}\left(\mathbb{R}, L^{2}\left(\Omega, \mathbb{C}^{6}\right)\right)$ and the above $H_{\varrho}\left(\mathbb{R}, L^{2}\left(\Omega, \mathbb{C}^{6}\right)\right)$-convergence for elements in $D\left(\partial_{0}\right)$. In fact we get

$$
\left(\partial_{0} M_{s}+N_{s}+A\right)^{-1} F \stackrel{s \rightarrow 0+}{\longrightarrow}\left(\partial_{0} M_{0}+N_{0}+A\right)^{-1} F \quad \text { for every } F \in H_{\varrho}\left(\mathbb{R}, L^{2}\left(\Omega, \mathbb{C}^{6}\right)\right)
$$

by the principle of uniform boundednes: 1 , i.e. strong convergence of the solution operators. In cases, where $\varepsilon_{0}$ vanishes, i.e. $\left.\varepsilon_{0}\right|_{L^{2}(\Omega)^{3}}=0$, we have the case of the eddy current approximation. We note that the usually considered case assumes $\Omega=\mathbb{R}^{3}$.

Let us conclude with some remarks:

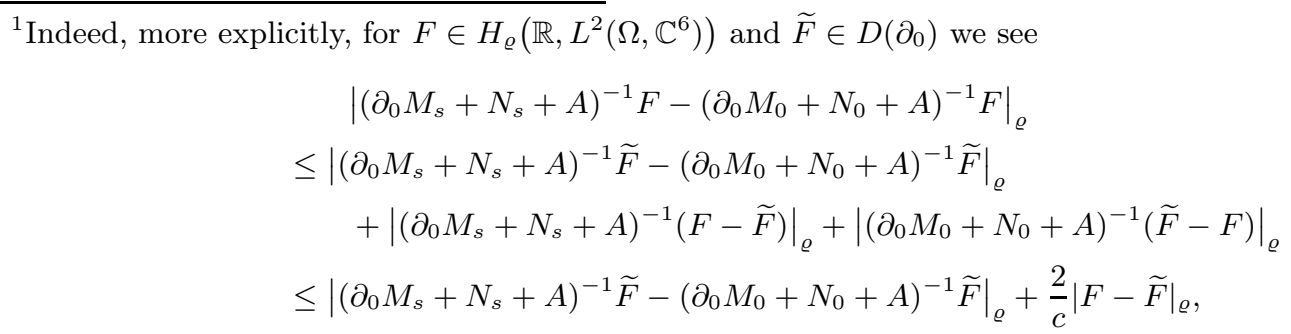

from which the desired convergence result follows by first choosing $\widetilde{F} \in D\left(\partial_{0}\right)$ to make the last term sufficiently small (independently of $s \in\left[0,1\left[\right.\right.$ ) and then, for this fixed choice of $\widetilde{F}$, we choose $\left.s_{0} \in\right] 0,1[$ sufficiently small to make the first term sufficiently small for all $s \in] 0, s_{0}[$. 
- The above rationale clearly also works for completely general sequences $\left(M_{s}\right)_{s},\left(N_{s}\right)_{s}$ with $M_{s}^{*}=M_{s}$ of continuous linear operators converging strongly to $M_{0}$ and $N_{0}$ with

$$
\varrho M_{s}+\mathfrak{R e} N_{s} \geq c>0
$$

for some $c \in] 0, \infty[$ and all $s \in[0,1[$. We have focused on the classical eddy current context to make the approach more tangible.

- Again we emphasize that our results depend

- neither on the size (bounded or unbounded)

- nor on the topology (genus, Betti-numbers)

- nor on the regularity (no regularity is assumed)

of the underlying domain resp. non-empty open set $\Omega$. Moreover, our methods extend immediately to domains resp. non-empty open sets $\Omega \subset \mathbb{R}^{N}, N \in \mathbb{N}$, or even to Riemannian manifolds $\Omega$ by replacing the curl-operators by the exterior resp. co-derivative.

- Our results remain valid even if mixed boundary conditions are considered. We just have to modify the skew-selfadjoint unbounded linear operator $A$ by

$$
A=\left(\begin{array}{cc}
0 & -\operatorname{corl}_{\Gamma_{1}}^{*} \\
\stackrel{\circ}{\operatorname{curl}_{\Gamma_{1}}} & 0
\end{array}\right)=\left(\begin{array}{cc}
0 & -\operatorname{curl}_{\Gamma_{2}} \\
\operatorname{corl}_{\Gamma_{1}} & 0
\end{array}\right) .
$$

Here the boundary $\Gamma:=\partial \Omega$ is decomposed into, let's say, two relative open disjoint subsets $\Gamma_{1} \neq \Gamma$ and $\Gamma_{2}:=\Gamma \backslash \overline{\Gamma_{1}}$. Following our definitions from above, we define $\operatorname{curl}_{\Gamma_{1}}$ as the closure in $L^{2}\left(\Omega, \mathbb{C}^{3}\right)$ of the curl-operator acting on the restrictions to $\Omega$ of $C_{1}\left(\mathbb{R}^{3}, \mathbb{C}^{3}\right)$-vector fields having compact support in $\mathbb{R}^{3}$ bounded away from the boundary part $\Gamma_{1}$ as well as

$$
\operatorname{curl}_{\Gamma_{2}}:=\operatorname{curr}_{\Gamma_{1}}^{*} .
$$

Once more, the structure of $A$ shows that $A$ is skew-selfadjoint.

- It is also clear that for uniform convergence of the coefficients we get uniform convergence of the solution operators in the sense that

$$
\partial_{0}^{-1}\left(\partial_{0} M_{s}+N_{s}+A\right)^{-1} \stackrel{s \rightarrow 0+}{\longrightarrow} \partial_{0}^{-1}\left(\partial_{0} M_{0}+N_{0}+A\right)^{-1}
$$

in $\mathcal{L}\left(H_{\varrho}\left(\mathbb{R}, L^{2}\left(\Omega, \mathbb{C}^{6}\right)\right), H_{\varrho}\left(\mathbb{R}, L^{2}\left(\Omega, \mathbb{C}^{6}\right)\right)\right)$.

\subsection{A Realistic Case}

For illustrational purposes we conclude with a realistic example, where the above limit situation occurs, e.g. an electromagnetic field in the presence of a laminated iron core in air (possibly with an air gap). Whereas in air the standard Maxwell equations are used, in the iron core the eddy current model is frequently assumed, see Figure 2.1. A possible, simple description would be that $\mu>0$ is a constant and $\varepsilon$ and $\sigma$ are piece-wise constant with

$$
\varepsilon= \begin{cases}\varepsilon_{\mathrm{air}} & \text { in air, } \\ \varepsilon_{\mathrm{lam}} & \text { in the insulating parts of the laminated iron core } \\ \varepsilon_{\mathrm{cor}} & \text { in the metal parts of the laminated iron core }\end{cases}
$$




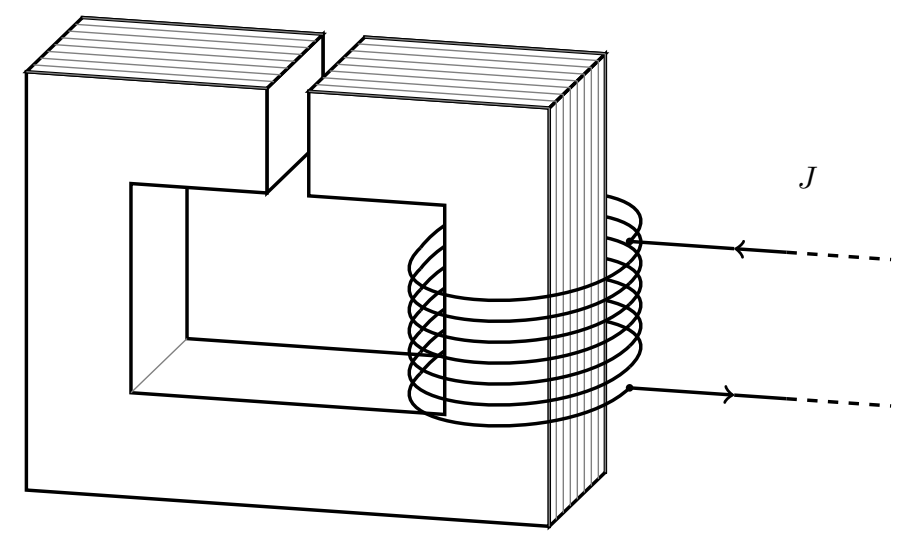

Figure 2.1: Laminated iron core in air.

and

$$
\sigma= \begin{cases}0 & \text { in air } \\ 0 & \text { in the insulating parts of the laminated iron core } \\ \sigma_{\text {cor }} & \text { in the metal parts of the laminated iron core }\end{cases}
$$

for $\varepsilon_{\text {air }}, \varepsilon_{\text {lam }}, \varepsilon_{\text {cor }}, \sigma_{\text {cor }}$ positive numbers. In the above we have established that replacing the - relative to $\sigma_{\text {cor }}$ - small value of the dielectricity $\varepsilon_{\text {cor }}$ can indeed be replaced by zero, i.e. $\varepsilon_{\text {cor }}=0$. In this situation the approximation result holds for any $\left.\varrho \in\right] 0, \infty[$.

\section{Acknowledgement}

We cordially thank Immanuel Anjam for creating the picture.

\section{References}

[1] Ana Alonso Rodríguez and Alberto Valli. Eddy current approximation of Maxwell equations. Theory, algorithms and applications. Milano: Springer, 2010.

[2] H. Ammari, A. Buffa, and J.-C. Nédélec. A justification of eddy currents model for the Maxwell equations. SIAM J. Appl. Math., 60(5):1805-1823, 2000.

[3] H. Ammari and J.-C. Nédélec. Low-frequency electromagnetic scattering. SIAM J. Math. Anal., 31(4):836-861, 2000.

[4] D. Pauly. Niederfrequenzasymptotik der Maxwell-Gleichung im inhomogenen und anisotropen Außengebiet. Dissertation, Universität Duisburg-Essen, Fakultät für Mathematik, http://duepublico.uni-duisburg-essen.de, 2003.

[5] D. Pauly. Complete low frequency asymptotics for time-harmonic generalized Maxwell equations in nonsmooth exterior domains. Asymptot. Anal., 60(3-4):125-184, 2008.

[6] T. Pepperl. Niederfrequenzasymptotik und Wirbelstrom-Approximation der verallgemeinerten dissipativen Maxwell-Gleichungen. Dissertation, Universität Duisburg-Essen, Fakultät für Mathematik, http://duepublico.uni-duisburg-essen.de, 2006. 
[7] R. Picard. On the low frequency asymptotics in electromagnetic theory. J. Reine Angew. Math., 354:50-73, 1984.

[8] R. Picard. An Elementary Hilbert Space Approach to Evolutionary Partial Differential Equations. Rend. Istit. Mat. Univ. Trieste, 42 suppl.:185-204, 2010.

[9] R. Picard and D. F. McGhee. Partial Differential Equations: A unified Hilbert Space Approach, volume 55 of De Gruyter Expositions in Mathematics. De Gruyter. Berlin, New York. 518 p., 2011.

[10] N. Weck and K.-J. Witsch. Complete low frequency analysis for the reduced wave equation with variable coefficients in three dimensions. Comm. Partial Differential Equations, 17(910):1619-1663, 1992.

[11] N. Weck and K.-J. Witsch. Generalized linear elasticity in exterior domains - I: Radiation problems. Math. Methods Appl. Sci., 20(17):1469-1500, 1997.

[12] N. Weck and K.-J. Witsch. Generalized linear elasticity in exterior domains - II: Lowfrequency asymptotics. Math. Methods Appl. Sci., 20(17):1501-1530, 1997. 